\title{
Automatic detection and counting of Callosobruchus maculatus (Fab.) Eggs on Vigna radiata seeds, using ImageJ
}

\author{
J. Georgina ${ }^{1 *}$, C. H. Arun ${ }^{2}$, M. Ramani Bai ${ }^{3}$ \\ ${ }^{1}$ Department of Zoology, Scott Christian College Autonomous, Nagercoil, Affliated to Manonmaniam Sundaranar University, Tirunelveli, Tamil Nadu, India. \\ ${ }^{2}$ Department of Computer Science, Nesamony Memorial Christian College, Marthandam, Tamil Nadu, India. \\ ${ }^{3}$ Department of Zoology, Muslim Arts College, Thiruvithancode, Tamil Nadu, India.
}

\section{ARTICLE INFO \\ Article history: \\ Received on: December 05, 2020 \\ Accepted on: February 02, 2021 \\ Available online: March 14, 2021}

\section{Key words:}

Callosobruchus maculatus,

Automated egg count,

Image processing,

Digital images,

Manual count.

\begin{abstract}
Callosobruchus maculatus is a major pest of stored pulses causing huge loss especially during the post-harvest period. Counting of obscure C. maculatus eggs performed in research studies are related to pest-control, pest-status, population demography, reproductive parameters, and also in sampling procedures. Counting of numerous tiny eggs is always performed manually which is laborious, time consuming, and tedious. Therefore, an efficient, automated egg counting of $C$. maculatus, was performed with image processing techniques using ImageJ software. Batch processing of 60 digital images was executed with inclusion of preprocessing, thresholding and filtering using Bandpass, Mexican hat, and Fast filters of ImageJ software. In terms of accuracy, Band-pass method performed best with a mean percentage error difference of 15.55, while Mexican hat and Fast filters recorded 25.66 and 32.41, respectively. Pearson's correlation was also highest (0.908) in Band-pass method. While comparing the execution time for the different methods, Fast Filter method showed highest percentage efficiency improvement of 65.53\%. Egg counting time was $852 \mathrm{~s}$ in case of manual count but in automated count with Band-pass, Mexican hat, and Fast Filters, it was 41, 32, and $13 \mathrm{~s}$, respectively. Laborious manual counting of C. maculatus eggs in future can be replaced by this automated procedure with good accuracy and rapid execution time.
\end{abstract}

\section{INTRODUCTION}

Pulses which are a rich source of protein are under much threat due to the infestation of pests, especially the Callosobruchus sps. Callosobruchus maculatus or the pulse beetle is a common pest which infests Vigna radiata or green gram pulse seeds in the fields but display an exponential growth, once moved to storage. The huge loss which they cause during post-harvest is substantially brought under control by the use of chemical pesticides which unfortunately pose a serious risk to the health of the user and consumer. An effective and safer alternative measure of control would be ideal and necessary.

Extensive research using various botanical products, radiations, and pheromones is performed on insects by researchers to determine their ovipositional inhibitory effects and these studies involve counting of eggs in the pre- and post-treatments [1-3]. Other research works involving egg counting of $C$. maculatus include factors affecting fecundity [4,5] and comparative study of ovipositional behavior between two species [6].

\section{*Corresponding Author:}

J. Georgina, Department of Zoology, Scott Christian College (Autonomous), Nagercoil, Affliated to Manonmaniam Sundaranar University,

Tirunelveli, Tamil Nadu, India.

E-mail:jgeorgina2018@gmail.com
Counting minute and numerous eggs manually during research is a tedious repetitive task which is time consuming and error-prone [7]. Eggs are always counted using the naked eye and sometimes with the aid of a magnifying lens. This standard method of manual count is error prone, time consuming, strains the eye, and limits the feasibility of large-scale experiments. The integration of computers and digital photography which are now used in many aspects of biology can certainly mitigate the problems during egg counting. Image processing techniques have wide range of applications in various fields and recently at a rapid rate in the field of biology. Fields of application include automated counts of bacterial colonies, cell counting, egg counting of amphibians and insects, larva counting, fisher ling counting, pollen grains, and other varied applications [8-12] from digital images.

Automated counts are much faster and accurate and reduces physical strain. In the present study, egg counting of C. maculatus is automated using the algorithms implemented in ImageJ, to develop a better, faster, efficient, and easy counting of eggs. Batch processing macros were developed for rapid counting of eggs from numerous images. There does not seem to be any previous work related to automated egg count of C. maculatus using image processing techniques. ImageJ is an open source image-processing program from the National Institutes of Health [13] and it is a valuable tool in research involving the analysis of digital images. 


\section{MATERIALS AND METHODS}

Green gram ( $V$. radiata) bought from the local market were analyzed for the presence of $C$. maculatus eggs on them. The egg laden seeds were separated manually and on hatching, the emerging adults were identified as C. maculatus using their unique features such as the large acute spine in the hind femur and subserrate antennae. The stock culture consisting of $1 \mathrm{~kg}$ of $V$. radiata seeds and the adult $C$. maculatus beetles were maintained at a room temperature of $28^{\circ} \pm 4^{\circ} \mathrm{C}$ until two generations of $C$. maculatus emerged. The egg-laden pulse seeds (415 days old) involved in ovipositional deterrence research work was used for image acquisition and egg counting.

\subsection{Image Acquisition}

The digital images of the pulses with opaque white eggs were captured using the microscope (Digital USB microscopic camera), by placing pulse seeds within a cavity of thin sponge that is wedged between two plain rectangular pieces of glasses. Elastic bands were used to bind both the glasses together and to keep the pulses secure, without any movements.

Twenty pulse seeds, mean length $\pm \mathrm{SD}(\mathrm{mm})=4.53 \pm 0.46$, were randomly taken from the stock culture and for each set of 20 pulses, digital images of both sides were taken by turning over the setup so as to increase the accuracy. Sixty digital images were created from random set of samples $(n=30)$ with 20 pulses each, taking into account both sides of a sample. A graph paper was stuck on the base stand of the microscopic camera so as to facilitate the setting up of the scale in the ImageJ program with proper resolution. ImageJ (v.1.51k) software program is used for automatic egg counting from the acquired digital images. Manual counting was performed by counting the eggs of the captured images with the naked eye.

\subsection{Image Processing in ImageJ}

Egg counting from the digital images using ImageJ (v.1.51k) was automated by executing three macros created with a sequence of image processing techniques [Figure 1]. In each of the macros, initially, the image is converted to 8 bit grey scale image (Image $\rightarrow 8$ bit). Preprocessing of images was done through selecting proper filters that would best facilitate subsequent thresholding measures. In the first macro, the image was pre-processed through the Band-pass filter (Image $\rightarrow$ Process $\rightarrow$ FFT $\rightarrow$ Band-pass filter) which has the ability to remove both high and low spatial frequencies in an image. The attributes in the Band-pass filter were set to filter large $=10$, filter small $=3$, suppression stripes are set to "none," tolerance of direction is set at $5 \%$ and autoscale set to "saturate." The threshold function was then used to binarize the image (Image $\rightarrow$ Adjust $\rightarrow$ Threshold), where the Renny Entropy threshold was selected and set threshold $(235,255)$ was specified after many trials to find the best fit. The final step is the counting of the eggs using the analyze particles function (Analyze $\rightarrow$ AnalyzeParticles) with size $=27-150$, circularity $=$ $0.70-1.00$ and show $=$ BareOutlines. The size and circularity were specified after adjustments to find the correct range in size.

In the second macro, after converting the image to 8 bit, the image was processed through Mexican Hat Filter (Plugins $\rightarrow$ Filters $\rightarrow$ Mexican hat filter), where the radius was set to 3 and then in the Threshold function setAutoThreshold $=0$ and DefaultDark $=0$ was selected. The Auto Threshold plugin ensures reproducibility and the deletion of user inconsistencies by the manual manipulation of thresholds.

The eggs were counted using the Analyzing Particles function with same settings as in the previous macro. In the case of Fast filter processing and

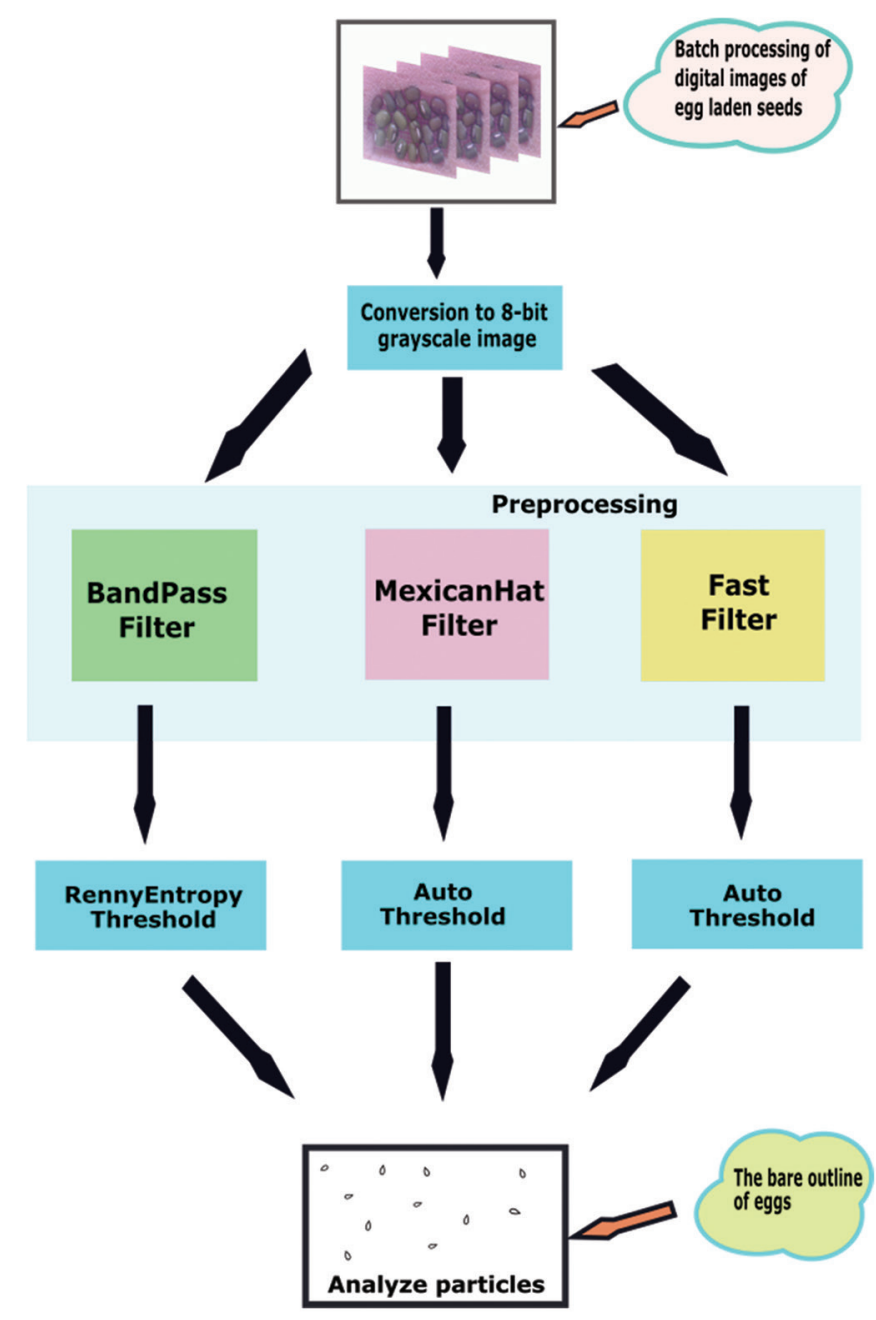

Figure 1: The overall flow chart of automatic egg count.

counting, after the image is converted to 8 bit, the image is processed using the Fast filter (Plugins $\rightarrow$ Filters $\rightarrow$ Fast filter), where the link filter is set to minimum, $\mathrm{x}=4, \mathrm{y}=4$, preprocessing $=$ smooth, and subtract offset $=20$. The setAutoThreshold was set to be "Default Dark," and then, the eggs were counted using Analyze Particles of same specifications. All of these processes are assembled as scripts in the macros for automatic analysis and batch processing. The macros were executed using the steps (Process $\rightarrow$ Batch $\rightarrow$ Macro) which save all the output files of the image, containing the egg bare outlines and the summary of each image with the count of the eggs. The manual count involved counting the eggs visually with the naked eye. The time taken for manual and automated counts of each method was also noted.

Mean percentage error difference was used to evaluate the accuracy of the automated counting in comparison with the manual counting method. Percentage error difference was calculated as (Automated egg count-Manual egg count)/Manual egg count $* 100$. Efficiency improvement (Manual egg count/Automated egg count) provides the efficiency of automated count over manual count in terms of execution time of the process.

\section{RESULTS AND DISCUSSION}

As shown in Table 1, the Pearson's correlation coefficient for the automated and manual egg count using the Band-pass filter method 
was 0.908 and the Cronbach's alpha of consistency was 0.951 . The Pearson's correlation coefficient for the automated and manual egg count using the Mexican hat filter method was 0.763 and the Cronbach's alpha measure of consistency was 0.863 . The Pearson's correlation coefficient for the automated and manual egg count using the Fast filter method was 0.368 and Cronbach's alpha measure of consistency was 0.538 . Hence, there is significant correlation with manual count and automated counts of the first two filter methods, but " $P$ " value for Fast filter with 0.045 indicates that the difference between both types of counts can be accepted and considered not significant. The scatter plot between the automated count with Band-pass [Figure 2a] and Mexican hat [Figure 2b] filters and manual egg counting shows high and moderate agreement with $\mathrm{R}^{[2]}$ value of 0.82 and 0.58 , respectively, while the scatter plot against Fast filter [Figure 2c] automated count and manual count illustrate very low agreement $(0.14)$ between both methods.

The percentage error difference between the manual count and the three methods of automated count shows high agreements between egg counting of Band-pass filter method and manual count as represented by the shorter box plot, as shown in Figure 3a. The line near the middle of box refers to the median and the top and bottom borders of the box are the $75^{\text {th }}$ and $25^{\text {th }}$ percentiles, respectively. The box plot of Mexican hat filter has much higher range of percentage error difference when compared with box plot of Band-pass filter. Fast filter box plot shows most widespread values and poor performance. Outliers are noted for both Mexican hat and Bandpass box plot. The bar graph [Figure 3b] illustrates the execution time of egg counting by the three filters in comparison with manual count.

Band-pass and Mexican filters of ImageJ program exhibited good results, showing similar level of performance with 12.07 and 12.63 mean egg count, respectively, when compared with manual count (11.03), while Fast filter mean egg count was 9.60 [Table 2]. Evaluation of time taken for execution gave an opposite result, where Fast filter's execution time was much less (13 s) when compared with Band-pass filter (41 s) and Mexican hat filter (32 s). Efficiency improvement values (Manual count time/automated count time) for Band-pass, Mexican hat, and Fast filter were 20.78, 26.62, and 65.53 , respectively. Fast filter recorded highest efficiency in terms of the time taken for execution. However, in spite of comparatively, lower efficiency in execution time Band-pass filter method provided best accuracy with lowest mean percentage error difference (15.55 \pm 2.27 ) and high correlation values [Table 1]. Mexican hat filter method was moderate in its accuracy with a mean percentage error

Table 1: Statistical analysis results showing the levels of correlation between the three automated egg counts and manual count and the mean percentage error difference.

\begin{tabular}{|c|c|c|c|c|c|}
\hline \multicolumn{6}{|c|}{ Paired samples correlations } \\
\hline Egg count pairs & Mean percentage error difference \pm SE & Pearson's & Critical $P$ value & $\mathbf{R}^{2}$ & Cronbach's alpha \\
\hline Band-pass filter & $15.55 \pm 2.27$ & 0.908 & 0.000 & 0.82 & 0.951 \\
\hline Mexican hat filter & $25.66 \pm 3.71$ & 0.763 & 0.000 & 0.58 & 0.863 \\
\hline Fast filter & $32.41 \pm 6.06$ & 0.368 & 0.045 & 0.14 & 0.538 \\
\hline
\end{tabular}

For Pearson's correlation at 0.05 level there is significant difference between the manual and all three methods of automated counts

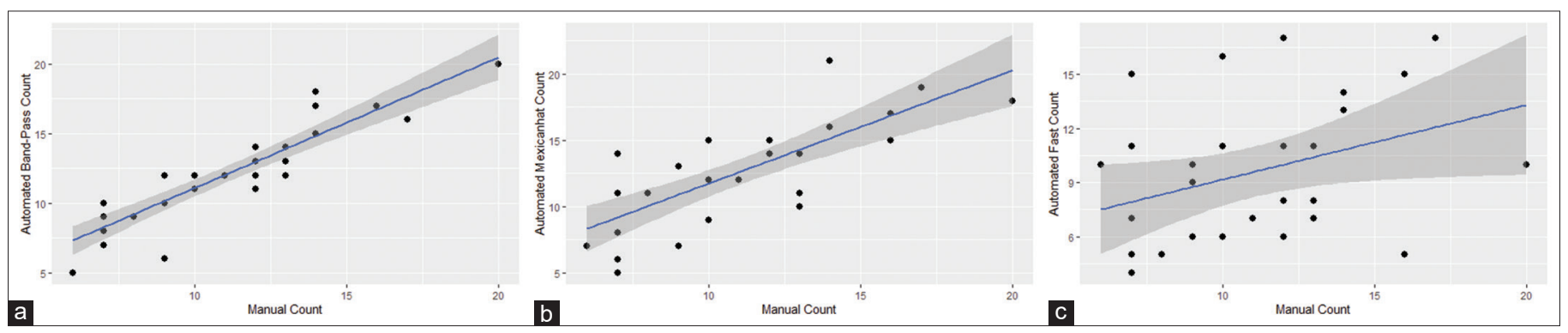

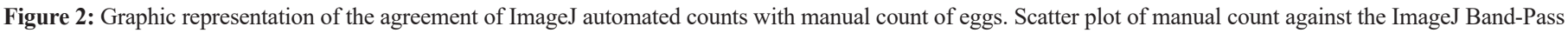
filter automated egg count showing good agreement $\left(\mathrm{R}^{2}=0.82\right)$, (b) scatter plot of manual count against the ImageJ Mexican hat filter automated egg count showing medium agreement $\left(\mathrm{R}^{2}=0.58\right)$, and $(\mathrm{c})$ scatter plot of manual count against the ImageJ Fast filter automated egg count showing very poor agreement $\left(\mathrm{R}^{2}=0.14\right)$.

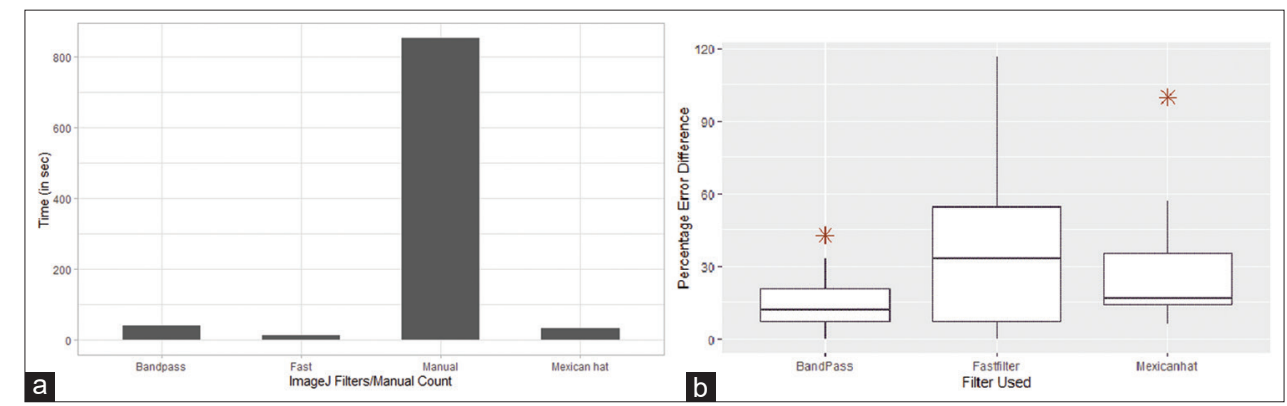

Figure 3: (a) Box plot showing the percentage error difference of the three filters with the manual count. Band pass filter of ImageJ performs best with least error difference and (b) bar graph showing the comparison of execution time in seconds by the various ImageJ filter methods and manual count. 
Table 2: The mean automated egg count, execution time, and efficiency improvement of the three filters - Band-pass filter, Mexican hat filter, and Fast filter.

\begin{tabular}{lccc}
\multicolumn{1}{c}{ Methods } & $\begin{array}{c}\text { Mean egg } \\
\text { count } \pm \text { SD }\end{array}$ & $\begin{array}{c}\text { Execution } \\
\text { time in s }\end{array}$ & $\begin{array}{c}\text { Efficiency } \\
\text { improvement \% }\end{array}$ \\
Band-pass filter & $12.07 \pm 3.64$ & 41 & 20.78 \\
Mexican hat filter & $12.63 \pm 3.94$ & 32 & 26.62 \\
Fast filter & $9.60 \pm 3.95$ & 13 & 65.53 \\
Manual count & $11.03 \pm 3.52$ & 852 & - \\
\hline
\end{tabular}

difference of $25.66 \pm 3.71$, while Fast filter recorded a value of $32.41 \pm 6.06$.

When manual and automated egg counts were compared that they seem to provide similar results except in Fast filter method showing deviation from the manual count. The mean percentage error difference for Band-pass filter was $15.55 \pm 2.27, \mathrm{R}^{[2]}$ value was 0.82 and Pearson's correlation coefficient value was 0.908 . On the other hand, there was a drastic difference in the execution time with automated counting of Fast filter, performing far too well. The execution time recorded for Fast filter method was $13 \mathrm{~s}$, while for Band-pass filter, it was 41 seconds. This study proves that automatic counting of $C$. maculatus eggs using ImageJ filters to be a quick, accurate, and non-destructive.

The Band-pass filter removes noise but preserves the larger aspects in the digital image [14], while Mexican hat filter separates signal from the noises using Laplacian or Gaussian filter. Manual and automated count have their own advantages and disadvantages. Magnified digital images provide a clearer sphere of egg detection, but the disadvantage to be taken into account are the unexposed eggs that might be found on the sides of pulses. Hand held manual counting involve direct counting with the naked eye or a magnifying lens which certainly is tedious, error prone, and time consuming.

An ideal condition for an automated count needs high contrast between the egg and the background and a low glare from the surface [15]. The challenges in this study of is the presence of white hilum on green gram seeds which are very similar to the eggs of $C$. maculatus. Furthermore, certain peak areas with highest reflection points on green gram may interfere, to give false results. Both these problems were eliminated using thresholding and circularity measure. The "threshold" command in ImageJ initially separates background from the particles of interest [16] automatically, and here, it separates the background from the white eggs, hilum region, and the glare points. The glare points and hilum are then distinguished from the egg by precise specification of the "circularity" measure. Since hilum has a greater circularity, than the egg their inclusion was eliminated from the count by specifying proper circularity $(0.70-1.00)$.

Manual method is extremely time consuming and quite impossible to be implemented in large-scale analyses, but still it is in usage due to its quality results and simple procedure [17]. The present study is promising by providing results with good accuracy in par with manual count. The correlation values and $\mathrm{R}^{[2]}$ values have shown the extent of good correlation between automated and manual count.

With regard to automatic egg counting, Band-pass and Mexican hat filters are superior to Fast filter of ImageJ. Furthermore, using macros and batch processing plugins of ImageJ program produced results at a very rapid rate which can never be achieved by manual or handheld egg count. In summary, the Band-pass filter coupled with Renyi
Entropy threshold and the other processes of ImageJ employed in this work have shown to be a good tool in automatic counting of C. maculatus eggs from digital images. In future, this work can be extended in counting insects, their eggs and also in distinguishing the eggs of early stages from eggs of later stages to assess their life cycle during infestation.

\section{CONCLUSION}

ImageJ software with its functionalities and filters aids well in counting the eggs of $C$. maculatus laid on $V$. radiata. Among the three filters of ImageJ software studied, Band-pass filter showed best performance with least error $(15.55 \pm 2.27)$. The time consuming and tedious job of manual egg counting is overcome by the batch processing of digital images which drastically reduce the time taken to count the eggs with high efficiency improvement (65.53). In future, the digital image processing techniques in ImageJ software would certainly prove to be efficient, especially the Band-pass filter with Renyi Entropy threshold, in counting the eggs of $C$. maculatus.

\section{AUTHOR CONTRIBUTIONS}

All authors made substantial contributions to conception and design, acquisition of data, or analysis and interpretation of data; took part in drafting the article or revising it critically for important intellectual content; agreed to submit to the current journal; gave final approval of the version to be published; and agree to be accountable for all aspects of the work. All the authors are eligible to be an author as per the international committee of medical journal editors (ICMJE) requirements/guidelines.

\section{FUNDING}

There is no funding to report.

\section{CONFLICTS OF INTEREST}

The authors report no financial or any other conflicts of interest in this work.

\section{PUBLISHER'S NOTE}

This journal remains neutral with regard to jurisdictional claims in published institutional affiliation.

\section{REFERENCES}

1. Stevenson PC, Green PW, Farrell IW, Brankin A, Mvumi BM, Belmain SR. Novel agmatine derivatives in Maerua edulis with bioactivity against Callosobruchus maculatus, a cosmopolitan storage insect pest. Front Plant Sci 2018;9:1506.

2. Yamane T, Goenaga J, Rönn JL, Arnqvist G. Male seminal fluid substances affect sperm competition success and female reproductive behavior in a seed beetle. PLoS One 2015;10:e0123770.

3. Mkenda PA, Stevenson PC, Ndakidemi P, Farman DI, Belmain SR. Contact and fumigant toxicity of five pesticidal plants against Callosobruchus maculatus (Coleoptera: Chrysomelidae) in stored cowpea (Vigna unguiculata). Int J Trop Insect Sci 2015;35:172-84.

4. Credland PF, Wright AW. Factors affecting female fecundity in the cowpea seed beetle, Callosobruchus maculatus (Coleoptera: Bruchidae). J Stored Prod Res 1989;25:125-36.

5. Wang MH, Horng SB. Egg dumping and life history strategy of Callosobruchus maculatus. Physiol Entomol 2004;29:26-31.

6. Giga DP, Canhão J. interference of oviposition behaviour in 
the cowpea weevils Callosobruchus rhodesianus (Pic) and Callosobruchus maculatus (F.). Int J Trop Insect Sci 1997;17:251-5.

7. Akintayo A, Tylka GL, Singh AK, Ganapathysubramanian B, Singh A, Sarkar S. A deep learning framework to discern and count microscopic nematode eggs. Sci Rep 2018;8:1-11.

8. Arena ET, Rueden CT, Hiner MC, Wang S, Yuan M, Eliceiri KW. Quantitating the cell: Turning images into numbers with ImageJ. Wiley Interdiscip Rev Dev Biol 2017;6:e260.

9. Nouhaud P, Mallard F, Poupardin R, Barghi N, Schlötterer C. High-throughput fecundity measurements in Drosophila. Sci Rep 2018;8:1-6.

10. Colas F, Tardivel M, Perchoc J, Lunven M, Forest B, Guyader G, et al. The ZooCAM, a new in-flow imaging system for fast onboard counting, sizing and classification of fish eggs and metazooplankton. Prog Oceanogr 2018;166:54-65.

11. Mussadiq Z, Laszlo B, Helyes L, Gyuricza C. Evaluation and comparison of open source program solutions for automatic seed counting on digital images. Comput Electron Agric 2015;117:194-9.

12. Fernandez-Gallego JA, Kefauver SC, Gutiérrez NA, NietoTaladriz MT, Araus JL. Wheat ear counting in-field conditions: High throughput and low-cost approach using RGB images. Plant Methods
2018;14:22.

13. Schneider CA, Rasband WS, Eliceiri KW. NIH Image to Image J: 25 years of image analysis. Nat Methods 2012;9:671-5.

14. Young K, Morrison H. Quantifying microglia morphology from photomicrographs of immunohistochemistry prepared tissue using ImageJ. J Vis Exp 2018;5:57648.

15. Karpova EK, Komyshev EG, Genaev MA, Adonyeva NV, Afonnikov DA, Eremina MA, et al. Quantifying Drosophila adults with the use of a smartphone. Biol Open 2020;9:bio054452.

16. Grishagin IV. Automatic cell counting with ImageJ. Anal Biochem 2015;473:63-5.

17. Cadena-Herrera D, Esparza-De Lara JE, Ramírez-Ibañez ND, López-Morales CA, Pérez NO, Flores-Ortiz LF, et al. Validation of three viable-cell counting methods: Manual, semi-automated, and automated. Biotechnol Rep 2015;1:9-16.

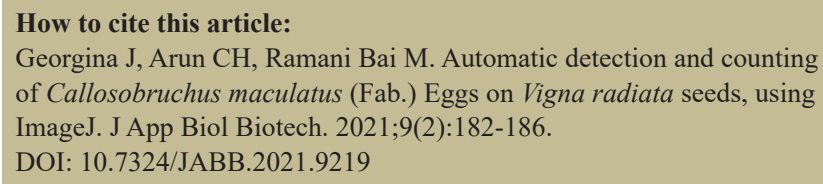

